\title{
Poster: Bluetooth Low Energy (BLE) Beacons Alone Didn't Work!
}

\author{
Yun Huang \\ SALT Lab \\ Syracuse University \\ Syracuse, NY 13244, USA \\ yhuang@syr.edu \\ Qunfang Wu \\ SALT Lab \\ Syracuse University \\ Syracuse, NY 13244, USA \\ qwu114@syr.edu \\ Yaxing Yao \\ SALT Lab \\ Syracuse University \\ Syracuse, NY 13244, USA \\ yyao08@syr.edu
}

Permission to make digital or hard copies of part or all of this work for personal or classroom use is granted without fee provided that copies are not made or distributed for profit or commercial advantage and that copies bear this notice and the full citation on the first page. Copyrights for third-party components of this work must be honored. For all other uses, contact the owner/author(s).

Copyright held by the owner/author(s).

UbiComp/ISWC'18 Adjunct, October 8-12, 2018, Singapore, Singapore

ACM 978-1-4503-5966-5/18/10.

https://doi.org/10.1145/3267305.3267634

\begin{abstract}
Bluetooth Low Energy (BLE) beacon technology is projected to be the leading proximity technology. Various business sectors are rapidly applying it because of its automatic location sensing capabilities, low cost and high accuracy. However, understanding of how people adopt beaconbased location sensing applications is still very limited. We developed a BLE beacon based system for automating class attendance taking. Our first field study with 42 students showed that about $38 \%$ of the students adopted it. Students had several misunderstandings and concerns of the technology that challenged its adoption. We revised the design by integrating a participatory sensing approach, where users can manually check in to their class by explicitly sharing their location using GPS. We conducted a second field study with 45 students under the same instructor in the following semester. The overall adoption of the attendance taking system was increased to $80 \%$.
\end{abstract}

\section{Author Keywords}

BLE beacon; Opportunistic sensing; participatory sensing; class attendance; location sharing.

\section{ACM Classification Keywords}

H.5.2 [Information interfaces and presentation (e.g., $\mathrm{HCl}$ )]:

User Interfaces 


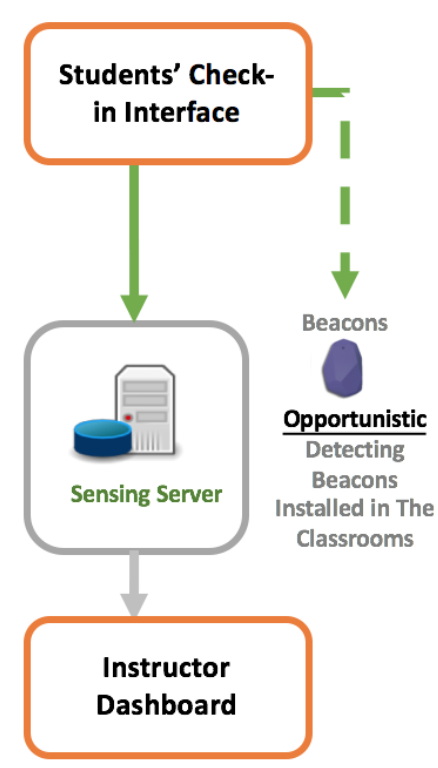

Figure 1: An illustration of the system model. Students need to turn on Bluetooth on their phones and share their location with the attendance app. When students enter the classroom where the beacon is installed, their attendance is automatically sent to the server. The instructor can check students' attendance records through a web-based dashboard.

\section{Introduction}

Bluetooth Low Energy (BLE) beacon technology enables location sensing with high accuracy and low cost $[1,6]$, thus it is increasingly used to automate location sensing (also called opportunistic sensing) [4, 3]. However, understanding of how people perceive and adopt this technology is still very limited, and there is a lack of empirical evidence that shares user experiences with this technology.

To gain a better understanding of this technology in the rea world, we developed a beacon-based location sensing app for taking class attendance. Our first round of study with a BLE beacon only approach failed; only $38 \%$ of 42 student users checked in automatically. Students had several mis understandings and concerns of the technology that challenged its adoption. We revised the design by integrating a participatory sensing approach, where users can share their location explicitly through GPS. Our second round of study with the combined solution successfully increased user's adoption with 45 students to $80 \%$ on average. We contribute (1) a system design of a hybrid sensing system where both opportunistic and participatory sensing approaches are applied; (2) empirical evidence of how BLE beacons alone failed with a low adoption rate and when a hybrid approach was needed.

\section{System Design}

Figure 1 illustrates the system model. To allow automatic check in with the beacon-based opportunistic sensing, students needed to turn on location sharing and Bluetooth on their mobile phones. Then when students enter the sensing area of the BLE beacon that is installed in the classroom, students can automatically check in. The second version of the design adds a manual check in feature on the student app interface. For the instructor, a web view was provided to review students' attendance records.

\section{First Field Study - BLE Beacon Only}

We conducted a first-round study with an undergraduate economic class in the 2017 Fall semester. The study goal was to evaluate how students would perceive and use the beacon-only app and the usability of the app in general. At the beginning of the study, two researchers went to the class and introduced the app to the students, demonstrating how to install, configure, and use the app. Then the instructor and students explored their own way of using the app for their classes. Students' participation was voluntary. Out of 69 enrolled students, 42 students installed and used the app. The app coverage started with $60 \%$ but gradually reduced in the following weeks. Overall, the average success rate is $38 \%$. After a month, most of the students stopped using the app.

We conducted semi-structure interviews with 13 students (six female and seven male) who participated in using the app. Interviews focused on how they used the app, if they had any concerns, and why they stopped using it. Each participant was paid $\$ 5$ for the study and the study was approved by our university's IRB.

All interviews were audio recorded and transcribed. We conducted a thematic analysis [2] that is commonly used to analyze qualitative data. Two researchers jointly coded two interview transcriptions at the sentence level which resulted in an initial code book. Then the two coders coded the rest of the interview transcriptions independently using the code book. When they had new codes that were not included by the initial code book, they added the new codes to their own copy of the code book.

The participants' feedback revealed several reasons why they had challenges using the beacon-only app, including misunderstandings of Bluetooth, concerns about location sharing, and confirmation accountability of opportunistic 
mobile sensing

Bluetooth Misunderstandings. First, the participants showed several misunderstandings of Bluetooth. For example, P8 thought turning on Bluetooth could consume cellular data from his cellphone data plan.

Bluetooth Misunderstandings "Normally I do not turn on Bluetooth, because it tends to lower my battery. I remember when we used to for attendance I only switched my Bluetooth on before the class. Sometimes I use it at home because I have a Bluetooth speakers but I usually have it switched off to save battery." (P4, female)

\section{Location Sharing Concerns}

"I don't want strange people

to message me on an open location, because it's mostly for hooking up. So I don't share my location always with an app." (P11, male)
Battery Consumption. Worrying about battery drainage was a common reason why participants turned off their Bluetooth, and as a result, failed to check in.

P6 and P11 thought that if Bluetooth was turned on, his personal data might be leaked. Due to these misunderstandings, many participants chose to turn off Bluetooth on their phones to avoid potential perceived risks. Since the opportunistic sensing in our app requires Bluetooth to be on, once it was turned off, the app would not be able to check the students in. Thus, they stopped using the app.

Location Sharing Concerns. To enable the opportunistic sensing check-in, users were required to "always" share the location on their mobile phones. However, this requirement was not clearly stated in the app. For example, P6 explained that she as well as many other users were not aware of such a requirement, thus they chose to share their location when the app was in use. However, this setting failed to check them in automatically. Moreover, some students thought that by "always" sharing their location, the app would be able to track their trajectory on campus, which might further cause unnecessary privacy issues. As P11 stated, he could potentially receive messages from random people based on his location.

Unfairness: Because of the above reasons, a number of students did not want to use the app for automatic checkin. Also, some participants mentioned that late students who did not use the app also received participation credit discouraged their continuing use of the app. From the instructor's perspective, he requested to have the flexibility to update late students' attendance to the system by himself, so that the late students can focus on the lecture.

In addition to the above issues, the "seamlessness" of beacon-based auto-location sensing seemed not always to be appreciated. Some participants wanted to be explicitly confirmed when they were automatically checked in.

Though there were challenges for some participants in adopting the technology, those who used the app, especially those who arrived early, liked the fact that the system showed the specific time they checked in to the class. They thought it would give the instructor a good impression.

\section{Second Design and Study - Hybrid Sensing}

Based on the findings from the first study, we made three revisions to the app. First, instead of relying on opportunistic sensing by using BLE beacons, we added a participatory sensing feature [5]-students can manually check in to the class when the auto-sensing feature fails or is not preferred. A manual check in has to be validated with the user's GPS location, which needs to be within several meters from the classroom location. Depending on the size of the classroom, the threshold value may vary. Regardless which check-in method is used, students will be informed if they have successfully checked in. Second, we clarified the misunderstandings of Bluetooth with pop-up messages when users first logged in to the app, declaring that the battery consumption of this Bluetooth app daily was no more than $2 \%$ based on our measurements, and that the app did not track students' location outside of the class. Third, we added another feature for instructors to update students' attendance for the students on the instructor website to address the instructor's request. 


\section{Unfairness}

"Whenever there's people

coming late in like 20 minutes before the class ends and signs

in, I kind of get angry because

I was here early for the partic-

ipation [credits], so it's kind of

unfair. " (P6, female)

"Yeah there were less and less people using it so he [the instructor] just took attendance regularly. There were people that came in late and they didn use the app. There would always be people whose app didn't work." (P11, male)

\section{Good Impression}

"I want credit for being early so it's nice for showing the arrival time. ... Yeah, I mean not that it makes a difference but at least Professor will acknowledge that l've been there since $12: 30$ when the class started at 12:45." (P1, female)
In order to evaluate if the hybrid sensing design can improve the adoption of the app, we conducted a second field study in Spring 2018 with the same instructor, however with another class of undergraduates. Out of 49 enrolled students, 45 students installed the app and used it throughout the semester.

The coverage of the hybrid sensing approach-including auto, manual-started from $67 \%$ and reached $95 \%$, with an average of $80 \%$; the coverage of the auto sensing alone started from $43 \%$ with an average of $59 \%$. On average, the instructor helped checked in 5 students each class. Given four students did not install the app, this suggested that when the instructor reviewed attendance record, on average, only one student failed to check in using the application.

\section{Conclusion and Future Work}

Our studies showed that applying a hybrid sensing approach successfully increased the adoption of a beaconbased application for taking class attendance. Future work will study if users persistently take one sensing approach or switch in between; what factors impact their decisions.

Acknowledgements: This research was supported by a Google Faculty Research Award. Also, this material is based upon work supported by the National Science Foundation under Grant No. \#1464312. Any opinions, findings, and conclusions or recommendations expressed in this material are those of the author(s) and do not necessarily reflect the views of the National Science Foundation.

\section{REFERENCES}

1. Mi-Young Bae and Dae-Jea Cho. 2015. Design and implementation of automatic attendance check system using BLE beacon. International Journal of Multimedia and Ubiquitous Engineering 10, 10 (2015), 177-186.

2. Richard E Boyatzis. 1998. Transforming qualitative information: Thematic analysis and code development. sage.

3. Diego Casado-Mansilla, Derek Foster, Shaun Lawson, Pablo Garaizar, and Diego López-de Ipiña. 2015. 'Close the Loop': An iBeacon App to Foster Recycling Through Just-in-Time Feedback. In Proceedings of the 33rd Annual ACM Conference Extended Abstracts on Human Factors in Computing Systems (CHI EA '15). ACM, New York, NY, USA, 1495-1500. DOI : http://dx.doi.org/10.1145/2702613.2732861

4. Andrea Corna, L Fontana, AA Nacci, and Donatella Sciuto. 2015. Occupancy detection via iBeacon on Android devices for smart building management. In Proceedings of the 2015 Design, Automation \& Test in Europe Conference \& Exhibition. EDA Consortium, 629-632.

5. Yun Huang, Alain Shema, and Huichuan Xia. 2017. A proposed genome of mobile and situated crowdsourcing and its design implications for encouraging contributions. International Journal of Human-Computer Studies 102 (2017), 69-80.

6. Shota Noguchi, Michitoshi Niibori, Erjing Zhou, and Masaru Kamada. 2015. Student attendance management system with bluetooth low energy beacon and android devices. In Network-Based Information Systems (NBiS), 2015 18th International Conference on. IEEE, 710-713. 